\title{
BRIGHT-RIMMED CLOUDS WITH IRAS POINT SOURCES: CANDIDATES FOR STAR FORMATION BY RADIATION-DRIVEN IMPLOSION
}

\author{
Koji Sugitani ${ }^{1.2}$, Yasuo Fukui ${ }^{3}$, And Katsuo Ogura ${ }^{4}$ \\ ${ }_{1}^{1}$. Physikalisches Institut. Universität zu Köln \\ ${ }^{2}$ College of General Education. Nagoya City University \\ ${ }^{3}$ Department of Astrophysics, Nagoya University \\ ${ }^{4}$ Kokugakuin University
}

We present preliminary results of a survey of bright-rimmed clouds associated with IRAS point sources.

Bright-rimmed globules associated with old HII regions have long been suspected as a potential site for star formation. Physical conditions of such clouds seem to well match to models of radiation-driven implosion, which have been studied as an effective process for induced star formation (e.g. Klein et al. 1985, Bertoldi 1989). Bright-rimmed globules associated with IRAS point sources are good candidates for the sites of induced star formation. Three well-established cases of radiation-driven implosions in brightrimmed globules (Ori I-2. IC1396-n, and L1206) were reported (Sugitani et al. 1989). Similar examples were also reported in HH46/47 (Olberg et al. 1989) and in GN21.38.9 (Duvert et al. 1990). However, the samples are still not numerous enough to establish comprehensive understanding of star formation by such implosion process.

We have surveyed for bright-rimmed clouds (cometary globules, and small clouds with curved bright rims) associated with IRAS point sources. The Palomar Sky Survey prints and IRAS Point Source Catalog were used for this survey. The surveyed regions are mainly around HII regions of Sharpless (1959) with texts of $\sim 60^{\prime}$ or larger. By using overlay maps showing IRAS point sources for the PSS prints, we selected the clouds having IRAS sources just surrounded by the curved rims. To exclude emission from diffuse dust, we included only the IRAS sources which are detected at $25 \mu \mathrm{m}$ and, at least, at one more band and have correlation coefficients of $F$ or better at $25 \mu \mathrm{m}$.

44 bright-rimmed clouds associated with IRAS sources have been selected from 18 HII regions. The most prominent region is S131(IC1396) having eleven bright-rimmed clouds. The second regions are S190 (IC1805) and S199 (IC1848), both of which have 5 clouds. Nine of the 44 clouds are associated with molecular outflows and one with a $\mathrm{HH}$ object, which are good signposts of young stellar objects. Most of their radii, R, determined by optical measurement are $\leqslant 0.5 \mathrm{pc}$, similar to those of Bok globules (Fig. 1). We estimated their masses by assuming a density of $3 \times 10^{4} \mathrm{~cm}^{-3}$. Most of the clouds have masses of $\sim 1-100 \mathbf{M}_{\odot}$, similar to Bok globules. We calculated IR luminosities of the IRAS sources from the IRAS $25 \mu \mathrm{m}$ fluxes (F.g. 2). The luminosities of the associated IRAS sources are relatively large, mostly $10-10^{3} \mathrm{~L}_{\odot}$, compared to those of the IRAS sources associated with dark globules or dense cores of dark cloud complexes (Beichman et al. 1986). This suggests that intermediate-mass stars are mainly formed in bright-rimmed clouds. On the other hand, the range of the luminosities is wide, $\sim 4$ $10^{5} \mathrm{~L}_{\odot}$, suggesting that relatively low-mass to high mass stars are formed in these bright- 
rimmed clouds under the influence of ionization-shock fronts. This fact appears to be consistent with the prediction of radiation-driven implosion models for star formation (e.g. Bedijin and Tenorio-Tagle 1984, and Klein et al. 1985). We estimated IRAS luminosity to cloud mass ratios following Sugitani et al. (1989). These ratios are significantly greater than that in dark globules or in dense cores of dark cloud complexes, suggesting more effective star formation in the bright-rimmed clouds.

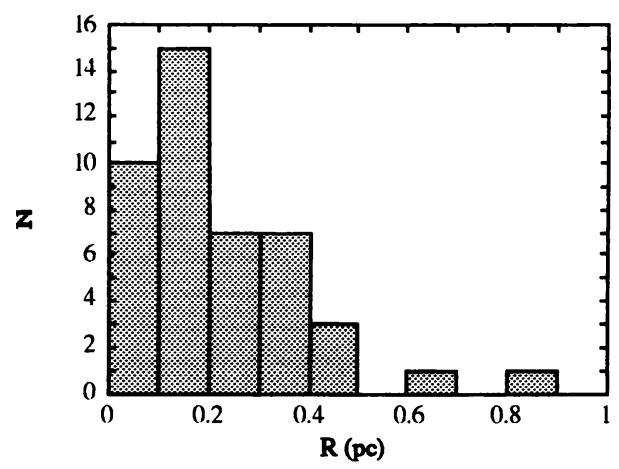

Fig. 1 --- Distribution of the cloud radii.

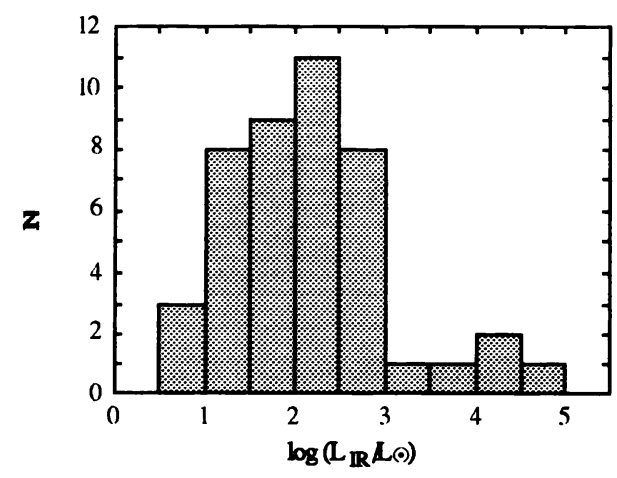

Fig. 2 --- Distribution of the luminosities of the IRAS point sources associated with the bright- rimmed clouds.

A detailed report of this survey will be presented elsewhere with a list and photographs of the bright-rimmed clouds.

\section{REFERENCES}

Bedijin, P. J., and Tenorio-Tagle, G. 1984, Astr. Ap., 135, 81.

Beicheman, C. A., Myers, P. C., Emerson, J. P., Haarris, S., Mathieu, R., Benson, P. J., Tennings, R. E. 1986, Ap. J., 307, 337.

Bertoldi, F. 1989, Ap. J., 346, 735.

Duvert, G., Gernicharo, J., Bachiller, R., and Gómez-González, J., Astr. Ap., 49, 57.

Klein, R. I., Sandford, M. T., and Whitaker, R. W. 1985, in Protostars and Planets II, ed.

D. C. Black and M. S. Mathews (Tucson: University of Arizona Press), p. 340.

Olberg, M., Reipurth, B., and Booth, R. S. 1989, in The Physics and Chemistry of Interstellar Molecular Clouds, ed. G. Winnewisser and J. T. Armstrong (Heidelberg: Springer-Verlag).

Sharpless, S. 1959, Ap. J. Suppl., 4, 257.

Sugitani, K., Fukui, Y., Mizuno, A, and Ohashi, N. 1989, Ap. J. (Letters), 342, L87. 